\title{
Fonction des discours dans les livres 6 et 7 des Histoires de Quinte-Curce
}

\section{Olivier Devillers}

\section{(2) OpenEdition \\ 1 Journals}

Édition électronique

URL : http://journals.openedition.org/rhetorique/256

DOI : 10.4000/rhetorique.256

ISSN : 2270-6909

Éditeur

UGA Éditions/Université Grenoble Alpes

Édition imprimée

ISBN : 978-2-84310-274-5

\section{Référence électronique}

Olivier Devillers, «Fonction des discours dans les livres 6 et 7 des Histoires de Quinte-Curce»,

Exercices de rhétorique [En ligne], 3 | 2014, mis en ligne le 16 juin 2014, consulté le 12 septembre 2020.

URL : http://journals.openedition.org/rhetorique/256 ; DOI : https://doi.org/10.4000/rhetorique.256

Ce document a été généré automatiquement le 12 septembre 2020.

\section{(c) (1) () (2)}

Les contenus de la revue Exercices de rhétorique sont mis à disposition selon les termes de la Licence Creative Commons Attribution - Pas d'Utilisation Commerciale - Partage dans les Mêmes Conditions 4.0 International. 


\title{
Fonction des discours dans les livres 6 et 7 des Histoires de Quinte-Curce
}

\author{
Olivier Devillers
}

1 L'objectif de cette contribution est d'explorer l'articulation entre deux observations souvent formulées à propos des Histoires de Quinte-Curce : la place qu'y occupent les discours, d'une part ${ }^{1}$; d'autre part la progression qu'y subit le personnage d'Alexandre, lequel se «barbarise » de plus en plus nettement à partir du livre $6^{2}$. Nous voudrions ainsi voir comment les discours reflètent à la fois cette barbarisation d'Alexandre et son isolement progressif dans la seconde pentade de l'ouvrage. Nous nous centrerons sur les discours des livres 6 et 7 qui se trouvent au début du processus de transformation du roi. Dans ce processus, le procès de Philotas, à la fin du livre 6, est tenu comme un moment-clés, mais dès le début du même livre, Quinte-Curce, évoquant le tournant du règne (6.2.1-4), parle $d$ '« une rancœur exprimée plus librement dans le cadre de plaintes mutuelles» (6.2.4: «liberior inter mutuas querelas dolor »). C'est de ce dialogue difficile que les discours discutés ici sont les témoins. Nous ne nous sommes néanmoins pas limité aux échanges entre Macédoniens ${ }^{4}$, car conformément à une pratique bien observée chez les historiens anciens ${ }^{5}$ - et à laquelle Quinte-Curce ne fait pas exception -, l'évocation des affaires extérieures se fait en écho aux affaires intérieures. Inversement, il a été nécessaire d'intégrer à la discussion certaines informations se trouvant dans les parties narratives. Cela a semblé d'autant plus justifié que, davantage que la construction rhétorique stricto sensu des discours, c'est leur fonction au service du propos général qui est au cœur du survol que nous proposons. Pratiquement, nous évoquerons les divers discours selon trois grands "moments » successifs. Nous reprendrons ensuite l'essentiel de nos remarques dans un propos dont l'orientation sera « transversale». 


\section{Tensions avec les troupes (6.2-6)}

1.1.

2 Alors qu'une rumeur annonce le retour de l'armée en Macédoine, créant une grande espérance dans le camp, Alexandre réagit en deux temps.

D’abord (6.2.18-19), « il se plaint » (« conquestus est ») auprès des préfets des troupes de la réaction de celles-ci. Dans cette brève intervention au style indirect, un thème principal est développé par le roi: la gloire, d'abord la sienne, qui semble sa préoccupation première (6.2.18: "ex medio gloriae spatio se reuocari ", " au milieu d'une carrière de gloire, on le rappelait en arrière ", trad. CUF), ensuite celle des troupes, qui sera plus grande encore si elles continuent à le suivre.

Ensuite, à l'instigation des préfets, il s'adresse aux troupes (6.3). Alors que le style indirect précédent a indiqué ce qu'il pense, ce style direct illustre sa stratégie en vue d'« apaiser les esprits par un discours lénifiant et de circonstance» (6.2.20: «animos eorum leni et apta oratione permulcere »). Il y emploie régulièrement la $1^{\mathrm{re}}$ pers. du pluriel pour souligner le chemin déjà parcouru ensemble, par lui et son armée (spéc. 6.3.3-6 ; $10-13$; 16-17). L'argumentation repose pour beaucoup sur l'interaction entre les notions de paix et d'empire (cf. 6 occ. d'« imperium ») : les soldats veulent la tranquillité, mais celle-ci ne sera accessible que si la domination sur l'ennemi est pleinement assurée, ce qu'en l'état, elle n'est pas ; il faut donc continuer la guerre. La gloire, déjà soulignée dans le style indirect précédent, vient ici en début et en fin de discours : dans les premières lignes, l'idée d'une "satietas gloriae » (6.3.1: "satiété de la gloire ») est associée au «desiderium pacis» ("l'aspiration à la paix ») que tout l'argumentaire qui suit rejette ; en particulier, Alexandre repousse pour lui-même la perspective - qu'il présente comme à la fois séduisante et impossible - d'une vie en Macédoine où il jouirait « d'une reconnaissance et d'une gloire acquise avec ses troupes» (6.3.5: «parta uobiscum laude et gloria »). Il nie ainsi que la gloire des Macédoniens puisse déjà se conjuguer au passé, et c'est naturellement qu'en fin d'exposé, il la projette dans le futur et fait miroiter au soldat le surcroît d'illustration qu'apporterait une victoire sur Bessus (6.3.17). De la sorte, il fait partager par ses troupes le désir d'une gloire nouvelle qui l'anime lui-même en tant que général. Pour ce faire, notamment, il travestit sa recherche d'une gloire personnelle en une gloire qui d'abord lui est commune avec les soldats (6.3.5: "uobiscum parta ", « acquise avec vous »), voire qui est propre à ceux-ci (6.3.17 : "gloriae uestrae ", "votre gloire »). Il en va de même du thème de la victoire, envisagé à la fois à la $1^{\text {re }}$ pers. du pluriel (6.3.17 : « in ipso limine uictoriae stamus », « nous nous trouvons au seuil même de la victoire ») et à la $2^{\mathrm{e}}$ pers. du pluriel (6.3.15: « uestrae [...] uictoriae », « votre victoire »).

\section{2.}

Un autre moment dans la relation avec les soldats prend place après le départ de Thalestris, la reine des Amazones ${ }^{6}$. Quinte-Curce - comme Diodore et Justin souligne en marge de cet épisode le tournant «oriental » pris par Alexandre, qui se comporte en monarque perse (6.6.1-87). Cela fait naître, parmi les anciens soldats de Philippe, des critiques qui sont produites au style indirect (6.6.9-10). Ces lignes renvoient au discours indirect dans lequel Alexandre se plaignait que ses troupes 
veuillent retourner en Macédoine (6.2.18-19). En effet, les soldats " renversent » pour ainsi dire le thème de la victoire tel qu'il y avait été évoqué par leur général. Tandis qu'Alexandre estimait que s'il rentrait de suite dans sa patrie, « il y ramènerait le destin d'un vaincu, et non d'un vainqueur " (6.2.19: «uicti magis quam uictoris fortunam in patriam relaturum »), les vétérans de Philippe considèrent que la honte serait de rentrer chez eux après s'être laissé corrompre par les mœurs perses et que déjà leur roi «davantage semblable aux vaincus qu'aux vainqueurs, s'était mué de général de la Macédoine en satrape de Darius " (6.6.10: "regem uictis quam uictoribus similiorem ex Macedoniae imperatore Darei satrapen factum »). On mesure le divorce entre Alexandre et ses hommes : pour le premier, c'est à écourter ses victoires qu'on prend le visage d'un vaincu; pour les seconds, c'est en prolongeant celles-ci jusqu'à adopter les coutumes des peuples conquis.

6 En l'occurrence, Alexandre ne répond pas en paroles, mais en actes, en faisant brûler le butin pris à l'ennemi, aussi bien ses effets personnels que ceux des soldats (6.6.15-16). Ce faisant, il abolit la distance qui le sépare des troupes en traitant identiquement leurs biens et les siens. Son action est efficace : l'armée cesse de maugréer et se réjouit que la discipline soit rétablie (6.6.17).

\section{Philotas (6.7-7.2)}

\section{1.}

7 L'« affaire Philotas » occupe la fin du livre 6, et ses prolongements le début du livre 7 ; le récit qu'en fait Quinte-Curce dépasse, par les détails fournis ainsi que par la dramatisation de la narration, ce qu'on trouve sur le même événement dans les autres sources $^{8}$. Alors qu'un complot contre Alexandre lui avait été dénoncé, Philotas n'en avait pas prévenu le roi, donnant prise au soupçon d'en être lui-même un instigateur. Quatre discours précèdent son arrestation. La plupart tournent autour du silence de Philotas qui n'a pas relayé auprès d'Alexandre la dénonciation qu'on lui avait faite : le Roi lui demande d'en rendre compte (6.7.31-32), lui-même s'en excuse (6.7.33-34), les Amis le trouvent suspect (6.8.10-14). Cela confirme la centralité d'Alexandre, «plaque tournante » de toute information. Ces discours semblent en outre donner au roi le rôle de juge unique. Tel est le rôle qu'il revendique (6.7.32: «fauentem habes iudicem », « tu as [en moi] un juge bien disposé à ton égard ») et que lui accordera Philotas (6.10.3). Tel est aussi ce qui ressort des propos de Cratère, le seul à ne pas revenir sur le silence de Philotas; considérant la culpabilité de celui-ci comme acquise, il pose uniquement la question des conséquences qu'aurait une éventuelle clémence d'Alexandre (6.8.4-9).

8 Ainsi, bien que l'affaire soit jugée par une assemblée de soldats, la seule qui était en principe habilitée à trancher (6.8.25), son issue paraît entièrement dépendre d'Alexandre. Le récit du procès le confirme en montrant d'une part l'impact du discours d'Alexandre sur les soldats, d'autre part le rôle déterminant que Philotas attribue luimême au roi, auquel il s'adresse à la $2^{\mathrm{e}}$ pers. du singulier, comme s'il était présent (9.10.11-15), alors même qu'il a déjà quitté l'assemblée (6.9.36 $)$. Quinte-Curce insiste de même sur le spectacle donné par Alexandre et Philotas. Le premier, dont le goût pour la mise en scène et l'autoreprésentation est bien souligné dans les Historiae ${ }^{10}$, se compose une attitude tragique, grave (6.9.1-2), et son propos est ponctué par les manifestations d'émoi de soldats qui le soutiennent $(6.9 .3 ; 9.6)$. Le second, pitoyable, enchaîné et défait 
(6.9.25-27; 9.32-33), plus semblable à un condamné qu'à un accusé (6.9.26 $\left.6^{11}\right)$, met fin à sa défense après avoir été interpellé par un soldat qui lui reproche de comploter contre ses bienfaiteurs (6.10.37).

9 Pour ce qui est des arguments, Alexandre souligne à nouveau l'étroitesse des liens qui l'unissent aux soldats ${ }^{12}$ : le thème se trouve au début (6.9.2), au milieu (6.9.12: « in uos liberos, parentes, consanguineos habeo », « en vous je tiens mes enfants, mes parents, mon sang ») et à la fin (6.9.24). Cette stratégie a pourtant ses limites: le praetor regius Amyntas, voulant surenchérir sur l'argument, s'écrie qu'Alexandre mort, les soldats n'auraient sans doute jamais revu leur patrie et leurs proches (6.9.28). La mention des femmes et de la patrie déplaît alors au roi qui sait qu'elle est de nature à démoraliser les troupes (6.9.29). Il s'était du reste, en 6.3, en mentionnant notamment les femmes et la patrie (6.3.5), efforcé de combattre cette forme de nostalgie. Un des arguments qu'il avait alors mis en avant était celui de la gloire $(6.3 .1 ; 3.5 ; 3.17)$. Il est le seul à citer celle-ci au cours de l'affaire Philotas. Il ne l'utilise toutefois plus pour se rapprocher de ses soldats, mais il accuse Philotas d'avoir été jaloux de sa gloire et d'avoir tenu des propos critiques après l'oracle de Jupiter Hammon (6.9.19: «haec sunt et iam pridem animi alienati a me et inuidentis gloriae meae indicia ", "voilà le fait d'un esprit qui à la fois s'est détourné depuis longtemps de moi et jalouse les manifestations de ma gloire ${ }^{13}$ "). Le roi apparaît dès lors sensible aux critiques dont il est objet - lesquelles semblent se fixer sur la question de la divinisation -, amorçant un mécanisme où la liberté de parole de ses proches se trouve réduite.

Philotas commence par souligner qu'il s'exprime alors qu'il est dans une situation inconfortable, puisqu'il est enchaîné (6.10.4); c'est aussi en mentionnant le climat hostile dans lequel il a présenté sa défense qu'il met un terme à celle-ci (6.10.37). Pour le reste, ses propos s'apparentent pour l'essentiel à une refutatio. On y retient le fait qu'il considère son sort comme lié à la volonté du seul roi, aussi bien pendant le procès (6.10.3 : « optimus causae meae iudex », " le meilleur juge de ma cause ») qu'avant celui-ci, lorsqu'il avait cru que le pardon qui lui avait dans un premier temps été accordé le mettait à l'abri (6.10.11-14 $\left.{ }^{14}\right)$, et la manière dont il évoque l'oracle d'Hammon, en réalisant combien sa franchise sur cette question lui avait été préjudiciable (6.10.26: « periculosa libertas », « une dangereuse liberté »).

11 Ensuite, Bolon s'en prend avec virulence à l'accusé (6.11.2-7). Il souligne comment Philotas se désolidarise des troupes macédoniennes, notamment en réquisitionnant des logements, en étalant ses richesses... Il ruine ainsi les efforts qu'avait faits l'accusé pour se gagner la sympathie des soldats, les appelant entre autres «commilitones» (6.10.8: " compagnons d'armes $\left.{ }^{15} »\right)$. Tel était sans doute aussi le but d'Alexandre en lui reprochant de ne pas s'adresser aux Macédoniens dans leur langue (6.9.34-36), un reproche qu'il a tenté de réfuter (6.10.23) et que reformule Bolon (6.11.4 $\left.{ }^{16}\right)$.

12 Soumis alors à la torture, Philotas passe aux aveux (6.10.21-30). Il fait remonter sa décision d'attenter à la vie d'Alexandre à des propos qu'Hégésiloque lui avait tenus ainsi qu'à son père Parménion après précisément l'oracle d'Hammon. À l'opposé du discours officiel d'Alexandre, le passage témoigne de ce que cette prétention à être fils de dieu a été vécue par certains comme une rupture avec le commun des mortels (6.11.24-25 : « amisimus Alexandrum, amisimus regem : incidimus in superbiam nec dis, quibus se exaequat, nes hominibus, quibus se eximit, tolerabilem. Nostrone sanguine deum fecimus qui nos fastidiat? qui grauetur mortalium adire concilium?" «Nous avons perdu Alexandre, nous avons perdu notre roi ; nous affrontons un orgueil qui n'est tolérable ni par les 
dieux, dont il se fait l'égal, ni par les hommes, dont il se retranche. Avons-nous au prix de notre sang créé un dieu qui nous méprise ? un dieu auquel il pèse de se rendre à une assemblée de mortels? ", trad. CUF modifiée).

\section{2.}

13 Après l'exécution de Philotas et en vue d'éteindre des propos séditieux (7.1.4), Alexandre met en accusation d'autres hommes.

14 Amyntas est ainsi accusé sous divers griefs, et notamment pour ne avoir pas épargné ses critiques au roi (7.1.16: "linguae uiolentiam temeritatemque uerborum ", " la violence du langage et la hardiesse des mots $»)$, ce qui semble un écho à ce que celui-ci dit de Philotas en 6.9.20 (" sed iam non uerba punienda sunt; linguae temeritas peruenit ad gladios ", « mais il ne s'agit pas aujourd'hui de punir des paroles; la hardiesse du langage en est venue aux épées »). Toutefois, Amyntas adopte à cet égard une attitude différente de Philotas, manifestant sa volonté de parler sans entraves et avec ses insignes d'écuyer (ce qu'il obtient, 7.1.18) et insistant sur le fait qu'il entend s'exprimer le corps et l'esprit libres (7.1.20). L'idée de liberté revient au demeurant à quelques reprises dans son propos (7.1.35: «liberi hominis », « un homme libre»; $38:$ :feci et liberius », «j'ai agi avec plus de liberté $»)$. Elle y côtoie d'autres thèmes récurrents: le danger ${ }^{17}$, le malheur ${ }^{18}$, la crainte $^{19}$, la nécessité de se justifier ${ }^{20}$ voire tout simplement le fait de prendre la parole ${ }^{21}$. L'interaction entre ces notions éclaire sur la question que devaient alors se poser les proches d'Alexandre : dans quelle mesure leur était-il encore possible de donner librement leur avis sans avoir à en rendre compte ou à redouter des périls? Par les mots « utinam Philotas quoque intra uerba peccasset » (7.1.25, « il aurait été mieux que Philotas s'en fût tenu à pécher par des propos»), Amyntas semble y répondre en signifiant que les paroles contre le roi sont moins graves que des actes. D'ailleurs, lorsqu'il donne un exemple de la versatilité et des exagérations verbales du soldat, Amyntas dit qu'à un instant, celui-ci désire affronter l'Inde et l'Océan et que, l'instant d'après, lui vient la nostalgie de ses enfants et de sa patrie (7.1.24). Or cette alternative est précisément celle qui est en jeu au début du livre 6, en particulier dans le discours qu'Alexandre tient en 6.3, et dont l'évocation maladroite par (apparemment un autre ${ }^{22}$ ) Amyntas avait suscité la colère du roi lors du procès de Philotas (6.9.28-29). Que Philotas ne craigne pas de s'y référer est un indice qu'il reste attaché à la liberté de langage qu'il défend. Pour ce qui est du reste de son argumentation, Amyntas se justifie d'avoir recherché l'amitié de Philotas en rappelant la faveur dont ce dernier jouissait auprès d'Alexandre lui-même (7.1.26-30); il s'agit là aussi d'un argument dont on trouve l'esquisse mutatis mutandis dans le discours de Philotas (6.10.24-25) et qui indique l'impact qu'avaient le roi et la vie de la cour sur les choix de chacun.

L'arrivée du frère cadet d'Amyntas donne lieu à deux courtes interventions, l'une de ce jeune homme (7.2.2), l'autre, à nouveau, d'Amyntas (7.2.6). Alexandre partage l'émoi de l'assemblée face à l'attitude de ce jeune garçon (7.2.6) et lui accorde son pardon ainsi qu'à ses frères. Il reprend alors deux des thèmes qu'avait privilégiés Amyntas: le danger (7.2.8: "periculi») et la nécessité de se justifier (7.2.9: «satius est purgatos esse quam suspectos", "mieux vaut s'être justifié que d'être suspect»). Mais tandis qu'Amyntas considérait qu'il n'aurait pas dû avoir à se défendre, Alexandre voit là un exercice propre à apporter l'apaisement : autre signe que le roi et les Macédoniens ne sont pas toujours sur la même longueur d'ondes. 

le roi, toujours attentif à se solidariser avec ses interlocuteurs, souligne que la trahison de Parménion les a tous affectés, et eux deux en particulier (7.2.13: « scelere Parmenionis omnes pariter adpetiti sumus, maxime ego ac tu», «nous avons tous été atteints par le crime de Parménion, surtout moi et toi »). D'autre part, Parménion, alors même qu'il ignore encore le sort qui lui est réservé, apprenant qu'Alexandre prépare une nouvelle expédition, évoque le thème de la gloire du roi en disant qu' « après tant d'exploits, il était temps que celui-ci songe à son salut » : "tempus saluti suae tanta iam parta gloria parcere» (7.2.24). La gloire d'Alexandre est évoquée ainsi comme une aspiration qui peut s'avérer nuisible à sa propre vie de roi. mentionner certains « qui se plaignirent librement de la mort de Parménion» (7.2.35: «quos libere mortem Parmenionis conquestos esse »). L'association de la liberté (« libere ») et des plaintes («conquestos esse») renvoie au début du livre 6, lorsque Quinte-Curce mentionne comme symptôme de la détérioration du règne d'Alexandre le «liberior inter mutuas querelas dolor » (6.2.4: « une rancœur exprimée plus librement dans le cadre de plaintes mutuelles"). Il en va de même des lignes où sont considérés ceux qui se plaignent avec trop de liberté (7.2.37 : "querentium »; « questi »; « libertatem linguae ») des conditions du service dans l'armée d'Alexandre. Les uns et les autres subissent des mesures discriminatoires de la part du roi.

\section{Opérations militaires (7.3-10)}

\section{1.}

Les opérations militaires qui suivent montrent qu'Alexandre met en pratique, par son comportement, l'idée de solidarité avec ses soldats dont il nourrit ses discours; il partage ainsi leurs épreuves lors de la traversée du pays des Parapamisades (spéc. 7.3.17) ou lorsqu'ils sont confrontés à la chaleur dans la partie désertique de la Sogdiane ( $c f$. son échange avec des éclaireurs en 7.5.10-12 ${ }^{23}$ ). Les Amis rappellent du reste que « son courage est l'unique remède pour son armée défaillante » (7.5.9: « animi sui magnitudinem unicum remedium deficientis exercitus esse »).

auparavant avait été reproduit le discours du Mède Cobarès à Bessus, des propos qui avaient été présentés comme s'affranchissant de la servilité, au prix même $\mathrm{du}$ danger (7.4.9). Effectivement, Bessus, sous l'emprise du vin, s'emporta contre Cobarès au point qu'il faillit le tuer. L'échange offre pour le monde barbare un parallèle à ce qui se passe à la cour d'Alexandre, où, comme l'a laissé entendre le discours d'Amyntas, la liberté de parole expose aux périls. Le rapprochement suggère la barbarisation d'Alexandre, ce que renforcent encore les ressemblances que l'on trouve entre cet épisode et le récit de la mort de Clitus au livre $8^{24}$.

Bessus, trahi par Spitaménès et livré au roi, se présente à ce dernier comme vengeur à la fois de Darius et d'Alexandre, qu'il met sur le même pied (7.5.37). Ce propos est de nature à donner du crédit à ceux qui reprochent à Alexandre de se barbariser. Les paroles prêtées un peu plus tard à des Barbares qui rangent Alexandre au nombre des dieux (7.6.6) vont dans le même sens. 


\section{2.}

21 La campagne contre les Scythes retient l'attention. Alexandre, blessé, et écarté du théâtre des combats, craint que les Macédoniens n'adhèrent pas à son dessein d'affronter ce peuple. Il s'adresse alors au cercle restreint des Amis et de quelques proches en vue de s'assurer leur soutien (7.7.10-19). On se trouve dans une configuration proche de celle qu'on observe au début du livre 6 , lorsqu'il recourt aux préfets pour disposer les soldats à continuer les conquêtes (6.2.18-19). Comme souvent lorsqu'il a besoin d'aide, il joue la carte de la solidarité, ainsi que le montrent 22 formes de la $1^{\text {re }}$ personne du pluriel (pronoms, possessifs, formes personnelles du verbe) dans la première partie de son adresse (7.7.11-17). Il y parle d'une gloire qui lui est commune avec les siens (7.7.13 : "gloriae nostrae »), met en avant le thème de leur victoire (7.7.12 : « inuictos »; « uictoribus »; 7.14 : « una uictoria »; 7.15 : « uicimus ») et, comme en $6.3^{25}$, il voit en celle-ci une arme propre à garantir la pérennité des conquêtes (spéc. 7.7.12). Vers la fin seulement, il en vient à lui-même, à sa blessure et à la perspective de sa mort, retrouvant un ton personnel (illustré par 10 formes de $1^{\text {re }}$ personne du singulier en 7.7.18-19) et une thématique qui renvoient au début de son discours : "discrimen me occupauit », « je suis devenu la proie du danger « (7.7.10).

Suit un échange entre Alexandre et le devin Aristandre qui avait révélé à Erygius que les entrailles étaient défavorables. Le roi reproche à Aristandre d'avoir divulgué le résultat de consultations privées ${ }^{26}$. Le devin apeuré «en perd presque la parole sous l'effet de la crainte»(7.7.26: "per metum etiam uoce suppressa»), faisant songer à Philotas qui avait éprouvé bien des peines à répondre au roi (6.9.33). Il commence par dire qu'il a indiqué " un danger ", "discrimen », utilisant le mot avec lequel Alexandre avait commencé son discours précédent (7.7.10), indice que l'homme, terrifié, essaie d'emblée de s'aligner sur le roi. Cette attitude en dit long sur la pression qu'il ressent et entrave sa liberté. Alexandre lui dit alors d'avoir confiance et que «les dieux lui [Alexandre] donnent la gloire en vue d'autres réalisations » (7.7.28: « ad alia sibi gloriam concedere deos »). Dans ce contexte privé, il mentionne une gloire qui lui est propre, ce qui marque une nuance avec ses discours en 6.3 et 7.7.10-18, lorsque, s'efforçant d'infléchir les réticences des Macédoniens, il évoque une gloire commune (cf. 6.3.5 et 17 ; 7.7.13). Finalement, Aristandre, après une nouvelle consultation des entrailles, trouve celles-ci particulièrement favorables.

L'épisode inclut aussi un assez long discours direct, que tient un ambassadeur scythe à Alexandre (7.8.12-30). En soulignant que ces propos émanent de Barbares, étrangers à la civilisation que représente Alexandre (spéc. 7.8.10-1127), Quinte-Curce invite à les considérer comme détachés des rapports de domination/servitude qui se nouent autour du roi et à y voir l'expression d'une forme de libertas. C'est aussi ce qu'entend le Scythe lorsqu'il évoque l'isolement de son peuple: "nec seruire ulli possumus, nec imperare desideramus" (7.8.16: "nous ne pouvons être esclaves de personne, ni ne regrettons d'en être les maîtres »). Par cet esprit de liberté, le Scythe fait penser au Mède Cobarès ${ }^{28}$. En tout cas, s'il rejette toute aspiration à l'imperium pour son peuple, l'ambassadeur scythe n'en sait pas moins combien cette préoccupation compte pour Alexandre. Dans la mesure où il revient sur ce thème, son discours peut tenir lieu de réponse à celui que le roi avait tenu en 6.3, dominé par les notions de paix et d'empire, d'autant que les deux textes partagent la même focalisation sur l'espace des conquêtes, se traduisant par l'énumération de peuples vaincus (ainsi 6.3.2-3 et 7.8.19). Ces 
conquêtes sont néanmoins perçues très différemment et le terme "imperium ", qui, chez Alexandre, suggère une autorité garante de pacification (spéc. 6.3 ; supra) ne figure pas ici, et le Scythe envisage plutôt l'action du Macédonien en termes de brigandage, avec l'emploi de termes comme "eripere» ou, bien sûr, "latro». Cette activité de brigand, du point de vue scythe, loin d'apporter la paix, génère des rancœurs inapaisables et une instabilité profonde. De même, alors que pour Alexandre, la victoire apporte l'obéissance des vaincus et la paix (7.7.12), pour le Scythe, elle lui apporte la guerre (7.8.21: "bellum tibi ex uictoria nascitur", "pour toi, la guerre vient de la victoire »). Il en va aussi de la sorte pour le thème de la gloire. Alexandre en 6.3 et en 7.7 mettait en avant une gloire qu'il avait en partage avec les Macédoniens. Le Scythe, au contraire, explique l'activité conquérante des Macédoniens par la seule cupidité d'Alexandre. Tandis que ce dernier invite ses soldats à ne pas éprouver de "satiété de la gloire» (6.3.1: "satietatem gloriae»), le Scythe parle des «mains insatiables » du roi (7.8.19: «insatiabiles manus »), d'une part en faisant allusion à une insatiabilité dans le désir de possession (et non de gloire), d'autre part en limitant cette insatiabilité au seul Alexandre (et en ne l'appliquant pas à ses soldats ${ }^{29}$ ), enfin en l'incarnant physiquement dans la personne du roi à travers l'image des mains. Cette dimension «physique » figure avant même le discours, lorsqu'il est dit que les Scythes s'étonnent de la taille modeste d'Alexandre $\left(7.8 .9^{30}\right)$, se prolonge dès la première phrase du Scythe à travers une image utilisant le corps - et les mains (7.8.11: «si di habitum corporis tui auiditati animi parem esse uoluissent, orbis te non caperet : altera manu Orientem, altera Occidentem contingeres", "si les dieux avaient voulu que ton physique fût à la taille de ta cupidité, le monde ne te suffirait pas : d'une main tu toucherais l'Orient, de l'autre l'Occident ", trad. CUF légèrement modifiée) - et revient dans le discours même (7.8.14: " fortunam tuam pressis manibus tene ", " tiens ta fortune à pleines mains ", trad. CUF). Alexandre apparait ainsi comme une incarnation de la convoitise, tandis que le reste des Macédoniens demeurent absents du discours du Scythe. Le roi a beau se présenter comme lié à ses troupes, pour le Scythe, il est le seul responsable des guerres qu'il mène. Le "ring composition » qui balise le discours conforte cette impression. Au début et à la fin est évoquée la rencontre de l'Orient et de l'Occident, de l'Asie et de l'Europe : d'une part, « altera manu Orientem, altera Occidentem contingeres» $(7.8 .12$; trad. supra), d'autre part, "nos et Asiae et Europae custodes habebis. Bactra, nisi diuidat Tanais, contingimus... » (7.8.30: «tu nous auras comme gardiens de l'Asie et de l'Europe. Nous touchons la Bactrie, si ce n'est que le Tanaïs nous en sépare... »). La récurrence du verbe « contingere » avec des sujets différents est significative : dans sa convoitise, Alexandre voudrait embrasser les deux continents (début du passage), mais dans les faits ce sont les Scythes qui les embrassent (fin du passage). Le constat comporte une menace : s'ils peuvent être gardiens, les Scythes peuvent aussi être ennemis et mettre en péril la Macédoine. Le désir personnel d'Alexandre de s'emparer sans cesse de territoires, loin de s'inscrire dans une logique de solidarité avec son peuple, peut alors conduire à un danger pour sa patrie. Quelles que soient les sources dont s'inspire Quinte-Curce, quels que soient les clichés qui infusent son argumentation ${ }^{31}$, il les utilise de manière à préciser son portrait d'Alexandre - en écho avec les discours précédents des livres 6 et 7.

24 Malgré ce discours qui invite à ne pas engager les hostilités, l'affrontement avec les Scythes a lieu. Alexandre n'a pas besoin de mots pour galvaniser ses troupes, dont il emporte l'adhésion en prenant part, malgré sa blessure, au combat (spéc. 7.9.11). La 
victoire est au rendez-vous et, comme le roi l'avait pensé, elle est gage de paix, puisqu'à sa nouvelle, l'Asie met fin à sa défection (7.9.17).

\section{3.}

Quelques péripéties précèdent la fin du livre. L'échange entre Alexandre et les nobles sogdiens (7.10.5-9), condamnés à mourir, mais qui finalement bénéficient de la clémence royale, montre sa capacité à mettre à son service personnel (quatre d'entre eux sont intégrés à ses gardes du corps) d'anciens ennemis de son armée.

Enfin, Alexandre met son honneur à s'emparer d'une citadelle réputée imprenable, que tenait le roi Arimaze. Celui-ci avait insolemment demandé à un ambassadeur envoyé par le roi «si Alexandre pouvait aussi voler» (7.11.5: « an Alexander etiam uolare posset »). Alexandre, quand ce mot lui est rapporté, se met en colère et déclare à ses conseillers qu'il allait faire en sorte qu'Arimaze " crût que les Macédoniens volaient aussi » (7.11.6: "ut crederet Macedones etiam uolare»). Il reprend alors les propos d'Arimaze en remplaçant "Alexander» par "Macedones". Ressentant les mots d'Arimaze comme un affront personnel, il décide de laver celui-ci, mais a besoin pour ce faire du concours des Macédoniens. C'est pourquoi il associe ces derniers à l'injure qui lui a été adressée: "indicaret insolentiam Barbari eludentis ipsos quia pinnas non haberent » (7.11.6: «il leur fit connaître l'insolence du Barbare qui se moquait d'eux parce qu'ils n'avaient pas d'ailes $\left.{ }^{32} »\right)$. Constituant alors un corps d'élite pour escalader des cimes dangereuses, il harangue ceux qui le composent ${ }^{33}$ en insistant sur ce qui les rapproche de lui : «o iuuenes et mei aequales» (7.11.8: " jeunes hommes qui êtes aussi mes contemporains»), "et mei documenta uobis dedi et uestra habeo» (7.11.8: "des preuves de l'homme que je suis, je vous en ai données, et j'en ai sur votre compte»), avant d'évoquer à la $1^{\text {re }}$ pers. du pluriel le thème de la victoire : «experiendo quae ceteri desperauerint, Asiam habemus in potestate» (7.11.10: "pour tenter là où tous les autres ont renoncé, nous avons l'Asie en notre pouvoir »). Même s'il se soucie sincèrement de ses hommes ${ }^{34}$, il tend à instrumentaliser le rapport privilégié qu'il entretient avec eux.

\section{Discours et narration}

Nos observations ont permis de mettre en avant certaines interactions entre discours et pas seulement entre discours qui se suivent; ainsi le discours du Scythe en 7.8 parait une réponse à celui d'Alexandre à ses proches en 7.7, mais aussi à celui qu'il a tenu à ses troupes en 6.3... On a pu aussi relever, pour les plus longs d'entre eux, une structure en ring composition, l'idée finale exprimée par l'orateur rappelant celle qu'il avait formulée au début de son propos. Mais c'est au niveau de la fonction des discours que nous nous situerons principalement.

\section{1.}

Si l'on s'en tient aux discours d'Alexandre et de ses proches, un de leurs buts récurrents est de manifester une solidarité entre les Macédoniens et le roi, ce que ce dernier manifeste non seulement dans ses propos, mais aussi dans ses actes, le plus souvent en partageant ostentatoirement les épreuves de ses soldats $(7.3 .17 ; 5.10-12 ; 9.11$; aussi 6.6.11-14). Dans ses discours, cela se traduit singulièrement par un recours à la $1^{\text {re }}$ pers. 
du pluriel qui le met sur le même pied que ses interlocuteurs, se présente pratiquement comme l'un d'entre eux et insiste sur les périls et les succès qu'ils ont partagés (6.3.1-4; 7.7.11-17). Simultanément, il développe un argumentaire qui implique tout particulièrement les notions de danger, de gloire et de victoire. Le danger vient d'ennemis qui sont en premier lieu sur le champ de bataille (Bessus en 6.3, les Scythes en 7.7), la gloire est partagée avec les soldats $(6.3 .5 ; 7.7 .13)$, la victoire est une arme de dissuasion qui incite les peuples étrangers à la paix (6.3.11; 7.7.12). Si des ennemis se manifestent sur le plan intérieur, la menace qu'ils font peser sur lui est présentée comme de nature à mettre en danger la collectivité entière (6.9.1-2, début du discours d'Alexandre contre Philotas), et ils sont à ce titre assimilables à des ennemis extérieurs (cf. 6.8.9, discours de Cratère).

Cette propagande se révèle généralement efficace auprès des troupes qu'Alexandre parvient à chaque fois à galvaniser, comme le soulignent les préfets en 6.2.21: " numquam infractos et abiectos recessisse, quotiens ipsius alacritatem et tanti animi spiritus haurire potuissent » («ils [les soldats] n'étaient jamais repartis démoralisés et abattus quand ils avaient eu la possibilité de goûter à son enthousiasme [d'Alexandre] et au souffle de son héroïsme ", trad. CUF modifiée ; aussi 6.4.1).

\section{2.}

C'est aux soldats de Philippe qu'il revient en 6.6.9-10 de "dévoiler" la rhétorique d'Alexandre : loin d'être leur égal, celui-ci s'éloigne de plus en plus d'eux, s'orientalise et se barbarise. Parallèlement, l'empire qu'il veut établir s'avère corrupteur et dangereux pour les valeurs macédoniennes (6.6.9-10). Les autres discours composés par Quinte-Curce vont dans ce même sens. C'est bien sûr le cas de ceux des ennemis, intérieurs et extérieurs, d'Alexandre. Mais les propos de ce dernier lui-même et de ses amis y concourent également, d'une manière plus indirecte toutefois.

Lors du procès de Philotas, la distance qui s'instaure entre Alexandre et certains des siens se cristallise autour de l'oracle d'Hammon ${ }^{35}$. Si, pour Alexandre, l'événement est le symbole de sa gloire, il est aux yeux de Philotas celui de son divorce avec les Macédoniens. Il s'ensuit une incompréhension - Alexandre voit une expression d'une inuidia, d'une jalousie, là où Philotas voit précisément le souci de le préserver de l' inuidia ${ }^{36}$ - qui fait qu'il devient difficile pour les Macédoniens de s'exprimer à ce sujet. La liberté de parole s'en trouve réduite, le climat politique détérioré et le danger d'une répression accru. C'est ce qu'indique, chez Philotas, la formule "periculosa libertas " (6.10.26: « une dangereuse liberté »), et l'interaction entre liberté de parole et danger est à nouveau implicite dans le discours d'Amyntas (7.1). Le thème est appelé à revenir dans le livre 8, dans la bouche de Clitus et d'Hermolaüs: eux aussi regrettent qu'Alexandre prétende avoir Zeus pour père $(8.1 .42 ; 7.13)$, et ils s'en prennent à la gloire du roi, qui l'amène à exposer la vie de ses soldats $(8.1 .29 ; 7.4$ et 11$)$. Le discours d'Alexandre sur une solidarité avec les siens est ainsi battu en brèche : sa gloire est source de danger à la fois pour ses proches, parce qu'ils ne peuvent pas en parler librement, et pour l'armée, parce que le roi s'engage dans des opérations militaires coûteuses en vies humaines. En principe, le danger vient des ennemis. En le faisant venir d'Alexandre, on l'associe à un ennemi extérieur, ce qui est en résonance avec le motif de sa barbarisation. 

Alexandre, ceux qui se soumettent à lui et l'acceptent comme souverain. L'ambassadeur scythe (7.8), issu d'une terre de confins davantage associée à un esprit de liberté (et donc assimilable à certains ennemis intérieurs), est exemplaire des premiers. Pour lui, Alexandre incarne une recherche personnelle de la gloire (incluant sa prétention à descendre d'un dieu ; 7.8.26) ; les victoires qu'il remporte sont l'aliment de la rancœur des vaincus et constituent une menace pour la paix de son peuple. Quant aux « soumis ", ils tendent de façon générale à voir en Alexandre leur seul interlocuteur, le dissociant de facto du reste de son peuple. Leur attitude étaie souvent indirectement la thèse d'une orientalisation : la continuité entre Darius et Alexandre est exprimée par Spitaménès (7.5.36), le roi est reconnu comme dieu par des ennemis (7.6.6).

qui appuient Alexandre adhèrent à sa vision des choses, comme Bolon, qui au cours du procès de Philotas, évoque « au premier degré » l'oracle d'Hammon (6.11.5). Toutefois, au cours du même procès, une intervention du préteur royal Amyntas, bien que destinée à accabler l'accusé, rappelle une divergence entre le roi et les soldats, l'un souhaitant la poursuite des conquêtes, les autres aspirant à retrouver leur patrie (6.9.28-29). Cela est le signe que les proches d'Alexandre peuvent, indirectement ou par maladresse, laisser subsister dans leurs propos des traits qui n'arrangent pas le roi. Notamment, en mettant Alexandre au cœur des débats (discours de Cratère), en insistant sur la dépendance des Macédoniens à son égard (7.5.9) ou en rappelant qu'il ne peut être traité comme le commun des mortels (6.8.14), ils trahissent la nature absolutiste d'un pouvoir qui, dans les faits, ne se partage pas. Nous compterons aussi parmi ces discours ceux d'Amyntas et de Parménion prononcés en des circonstances où ils ménagent leurs critiques à Alexandre. Eux aussi laissent apparaître que le danger pour le roi et les Macédoniens ne vient pas seulement des ennemis, extérieurs ou intérieurs, mais du roi lui-même. Ainsi Parménion semble regretter qu'en vue d'accroître son illustration, celui-ci s'expose inconsidérément (7.2.24), une réflexion qui sera reprise par les Amis, notamment après qu'Alexandre se sera mis en danger lors du siège des Malliens (9.4.30-5.13). Amyntas souligne que la structure centralisatrice de la cour, avec des favoris auxquels il faut plaire, puis qui tombent en disgrâce, est une source de dangers pour la noblesse (7.1.29: «tu, hercules, si uerum audire uis, rex, huius nobis periculi es causa ", " c'est toi, par Hercule, si tu veux entendre la vérité, roi, qui es la cause de ce danger qui pèse sur moi ${ }^{37} »$ ).

Enfin, pour ce qui est d'Alexandre lui-même, les circonstances de ses discours jettent souvent un doute sur sa sincéritée ${ }^{38}$. En 6.3 , il parle après que les préfets l'ont explicitement engagé à tenir aux troupes un discours qui puisse les calmer; lors du procès de Philotas, il lui faut mettre de son côté l'armée qui a apparemment le dernier mot dans ce type d'affaires capitales (6.8.25); au moment d'engager le combat contre les Scythes, il veut être sûr que la défection de la Bactriane et son mauvais état de santé n'ont pas un effet démoralisateur sur les troupes (7.7.6-7). Ailleurs, il cherche à obtenir le concours de ses interlocuteurs, ainsi l'exécuteur de Parménion (7.2.13) ou les combattants envoyés sur la roche d'Arimaze (7.11.8). Le soupçon existe donc que sa véritable pensée ne corresponde pas à ces propos fabriqués pour convaincre et effectivement il laisse parfois échapper les signes d'une autre manière de voir les faits. Durant le procès de Philotas en particulier ${ }^{39}$, il exprime une susceptibilité sur le sujet de sa propre gloire, autour notamment de la question de son origine divine, qui le conduit à sévir contre ceux qui lui en semblent jaloux. Il y conjugue le thème de la victoire à la

Exercices de rhétorique, 3 | 2014 
$1^{\text {re }}$ pers. du singulier (6.9.21: " meam uictoriam », « ma victoire »), tout comme, dans le contexte privé de relations avec son devin Aristandre, il évoque une gloire qui lui est propre (7.7.28). Ce souci de son illustration, devenu orgueil, constitue un danger pour ses proches, ce que met en avant Amyntas et qui se traduira au livre 8 par le meurtre de Clitus. Sa conception de la libertas paraît également assez restrictive. Déjà lors du procès de Philotas, il semble estimer qu'il n'aurait pas été déplacé de le punir pour ses propos, même s'il ne l'a pas fait $\left(6.9 .20^{40}\right)$. De même, bien qu'il pardonne à Amyntas, il ne s'excuse pas pour le danger qu'il lui a fait courir, mais estime salutaire de l'avoir ainsi amené à se justifier (7.2.9). Aussi n'hésite-t-il pas à exercer une pression sur les siens ; le devin Aristandre est visiblement intimidé (7.7.26), ceux qui se plaignent après la mort de Parménion endurent des mesures discriminatoires (7.2.35-37). Les discours $\mathrm{du}$ roi laissent donc, en arrière-plan de leur habillage rhétorique et propagandiste, percevoir une vision et une pratique absolutistes du pouvoir. Sur ce point, les discours des livres ultérieurs marqueront encore une progression ${ }^{41}$.

En somme, les discours que nous avons évoqués semblent renvoyer à deux plans narratifs : d'un côté, l'affirmation par Alexandre de sa solidarité avec son peuple, se traduisant par l'adhésion et la participation de celui-ci à ses entreprises; de l'autre, des éléments qui mettent en évidence la distance croissante qui, du fait des circonstances et de sa volonté propre, s'installe entre le roi et les siens ${ }^{42}$. Cela traduit l'intrication, dans la seconde pentade des Histoires, de deux « intrigues ", l'une qui relate la réussite objective, implacable, d'une conquête, l'autre qui laisse entendre la transformation irréversible d'une royauté en tyrannie ${ }^{43}$.

Nous ajouterons deux remarques. D'une part, la capacité de Quinte-Curce à mettre les mêmes thèmes (victoire, gloire, danger...) dans la bouche de plusieurs personnages qui jettent sur eux des éclairages différents selon une construction « thèse » (un roi écouté) / « antithèse » (un roi contesté), dont la narration des Historiae opérerait la « synthèse » (réussite militaire, échec politique), paraît profondément marquée par l'enseignement qui était fait de la rhétorique à Rome ${ }^{44}$. D'autre part, les relations de pouvoir qui sont au cœur des discours, qu'il s'agisse de la question de l'impérialisme, comme le montre le discours du Scythe ${ }^{45}$, ou sur le plan intérieur, du rapport entre le souverain et ses sujets, comme l'indique la familiarité entre le discours d'Amyntas et celui de Terentius dans les Annales de Tacite (Ann., 6.846), trouvent assurément un écho dans les préoccupations des Romains du premier siècle de l'Empire, confirmant le Principat romain comme l'horizon idéologique des Historiae Alexandri ${ }^{47}$.

\section{NOTES}

1. Par exemple F. Helmreich, Die Reden bei Curtius, Paderborn, F. Schöning, 1927. Aussi

F. Minissale, Curzio Rufo, un romanziere della Storia, Messine, Peloritana Editrice, 1983, p. 44-57 ;

E. Baynham, Alexander the Great. The Unique History of Quintus Curtius, Ann Arbor, Univ. of Michigan Press, 1998, p. 46-56.

2. Spéc. E. Baynham, op.cit. Aussi D. Spencer, «Perspective and Poetics in Curtius' Gorgeous East ", Acta Classica, 48, 2005, p. 121-140 (spéc. p. 132). 
3. I. Yakoubovitch, «La mise en scène du pouvoir chez Quinte-Curce (VIII-X) », Vita Latina, 180, 2009, p. 27 ; aussi R. Rollinger, « Die Philotas-Affär, Alexander III, und die Bedeutung der Deixiosis im Werk des Q. Curtius Rufus », Gymnasium, 116 (3), p. 2009, p. 266.

4. Nous avons toutefois écarté quelques interventions courtes qui nous ont semblé peu significatives : 6.4.8-13; 6.5.3; 7.4.4-6; 7.6.15.

5. Par ex. O. Devillers, « Rome et les provinces. Analogies, transferts, interactions dans l'Agricola et dans les Annales", à paraître dans O. Devillers éd., Les opera minora et le développement de l'historiographie tacitéenne, Bordeaux, Ausonius Éditions.

6. Sur la réélaboration de l'épisode par Quinte-Curce, en fonction d'une mise en évidence de l'orientalisation d'Alexandre, M. Munding, "Alexander and the Amazon Queen », Graeco-Latina Brunensia, 16 (2), 2011, spéc. p. 137-142. Dans une perspective plus générale, E. Baynham, "Alexander and the Amazons ", Classical Quarterly, 51 (1), 2001, p. 115-126. Aussi M. Daumas, "Alexandre et la reine des Amazones ", Revue des Études Anciennes, 94, 1992, p. 347-354.

7. M. Daumas, op. cit., p. 347, parle à propos de la venue de Thalestris d'une «fable moralisatrice destinée à mettre en garde les Grecs contre les dangers de l'Orient » (aussi p. 353).

8. Par ex. R. Rollinger, op. cit., p. 268 ; E. Kapetanopoulos, "Alexander's 'patrius sermo'in the Philotas Affair ", Ancient World, 30 (2), 1999, p. 118-121. Sur le rôle des discours judicaires rapportés dans le passage au service de cette dramatisation, Baynham 1998, 47 ; brièvement J. Marincola, «Speeches in Classical Historiography », dans J. Marincola éd., A Companion to Greek and Roman Historiography, Malden-Oxford-Carlton, Blackwell Publishing, 2007, p. 128.

9. En 6.10.32, il s'adresse également à la $2^{\mathrm{e}}$ pers. à son père Parménion, lui aussi absent.

10. Par ex. I. Yakoubovitch, op. cit., p. 24-32 (ainsi p. 25 : «Il cherche donc à promouvoir l'image idéale d'un roi grand général, proche de ses hommes... »).

11. Aussi 6.10.3 (propos de Philotas) : « a praesente damnatus sum » («j'ai été contenté alors qu'il [Alexandre] était présent ») ; 11.15 : « utpote et damnatus » (« comme un condamné »).

12. Cf. J.A.P. Gissel, «The Philotas Affair in Curtius Account of Alexander (VI.7-11). A Rhetorical Analysis », Classica \& Mediaevalia, 46, 1995, p. 226, 229.

13. Sur l'oracle d'Hammon, 4.7. Cf. E.A. Fredericksmeyer, "Alexander, Zeus Ammon and the Conquest of Asia ", Transactions and Proceedings of the American Philological Association, 121, 1991, p. 199-214.

14. Le geste même par lequel lui est accordé ce pardon - Alexandre lui tend la main droite en gage de réconciliation - a une forte connotation orientale et le fait qu'il soit signalé avec quelque insistance $(6.7 .35 ; 10.11 ; 10.14)$ est l'indice de l'orientalisation du gouvernement d'Alexandre ; R. Rollinger, op. cit., p. 257-273.

15. Aussi 6.10.24: «nobis », $1^{\text {re }}$ pers. du pluriel.

16. E. Kapetanopoulos, op. cit., p.117-128, y voit un élément de dramatisation introduit par Quinte-Curce.

17. 7.1.21: «periculum »; $28:$ «periculi »; $37:$ : periculosius».

18. 7.1.19: «tristiorem »; $23:$ : tristius ».

19. 7.1.20: «timere $» ; 28:$ : timere »; $36:$ «metus».

20. 7.1.21: « defendere »; $21:$ « purgari »; $26:$ « eo infitias »; $31:$ «purgare »; « diluet crimen »; 32 : «purgare»; $35:$ : infitias eo »; « excusem »; $37:$ « defensionem ».

21. 7.1.19: « confitemur »; 20 : « dicimus »; 21 : « sermonis »; «dicerem »; « dicta »; « oratione »; 22 : « uox»; 24 : « uituperamus », « laudamus », « miseremur », « irascimur»; $25:$ « conloquentium uoces » ; « uerba »; 31 : « locutus est »; 35 : « locutum esse »; « uerba »; $37:$ : tacere quam dicere »; «dicturus sum».

22. Pour W. Heckel, « Amyntas, Son of Andromenes », Greek, Roman and Byzantine Studies, 16, 1975, p. 395-397, il s'agirait du même homme (aussi « Notes on Q. Curtius Rufus'History of Alexander », Acta Classica, 37, 1994, p. 69-70).

23. Sur sa sollicitude pour les troupes, aussi 7.5.16. 
24. E. Baynham, op. cit., p. 53-54.

25. Cf. similitude entre 7.7.15: "at hercule si paulum cessauerimus, in tergis nostris Scythi haerebunt ", "par Hercule, si nous venons à nous relâcher, nous aurons les Scythes sur le dos ", et 6.3.10 : « omnes hi simul terga nostra uidebunt, insequentur ", "tous ceux-ci, dès qu'ils nous verront le dos tourné, se mettront à notre chasse ».

26. Cf. 7.7.24: « arcana mea et secreta », « mes secrets privés »; cette idée de secret se retrouve un peu plus loin, lorsqu'Alexandre décide de ne pas divulguer un revers essuyé face à Spitaménès (7.7.39).

27. Cette image des Scythes viendrait d'Hérodote; $c f$. F. Hartog, Le Miroir d'Hérodote : essai sur la représentation de l'autre, Paris, Gallimard, 1980 (nouvelles éd. 1991 et 2001).

28. Les discours des deux hommes ont aussi en commun le recours à des aphorismes locaux (7.4.13; 8.25) ; E. Baynham, op. cit., p. 53.

29. Aussi 7.8.20: "Primus omnium satietate parasti famem », «le premier de tous, la satiété t'a apporté l'appétit ».

30. Le trait se trouve aussi à propos de la reine des Amazones en 6.5.29; voir les remarques de E. Baynham, op. cit., p. $117 \mathrm{n}^{\circ} 15$.

31. Tels sont les deux aspects que privilégie L. Ballesteros-Pastor, "Le discours du Scythe à Alexandre le Grand (Quinte-Curce 7.8.12-30) », Rheinisches Museum für Philologie, 146, 2003, p. 23-37.

32. L'écho fidèle aux paroles d'Arimaze aurait été : « eludentis ipsum quia pinnas non haberet ».

33. Pour E. Baynham, op.cit., p.52, ce discours figure parmi ceux que Quinte-Curce aurait pu créer de toutes pièces.

34. Cf. 7.11.20: "rex non cupidine magis potiundi loci quam uice eorum quos ad tam manifestum periculum miserat », «le roi moins tourmenté par le désir de s'emparer de la position que par le sort de ceux qu'il avait envoyés à un péril si évident »; F. Ripoll, «La prise du rocher d'Aornos chez Quinte-Curce (VIII, 11): déformation historique, transposition épique, démonstration morale », Vita Latina, 180, 2009, p. 21.

35. Sur le rôle de ce thème dans la critique d'Alexandre, J.A.P. Gissel, op. cit., p. 228.

36. Cf. l'écho entre 6.9.19: « inuidentis » (« un jaloux»), dans les propos d'Alexandre, et 6.10.27: « non enim faciebam inuidiam, sed pro eo timebam » («je ne cherchais à faire naître aucune jalousie, mais craignais pour lui »), dans les propos de Philotas.

37. Amyntas voit également Alexandre comme cause du péril auquel il a été exposé à la fin de son discours, mais dans une autre perspective (7.1.40, en rapport avec une mission auprès d'Olympias).

38. Ainsi I. Yakoubovitch, op. cit., p. $26:$ « insensiblement [...] Alexandre glisse de la mise en scène à la manipulation, voire à la machination. »

39. E. Baynham, op. cit., p. 48, épingle notamment la différence entre les discours d'Alexandre en 6.3 et en 6.9 (procès de Philotas).

40. Amyntas semble au contraire penser que tant qu'il s'en tenait à des propos, Philotas n'était pas punissable : 7.1.25: « utinam Philotas quoque intra uerba peccasset » (« il aurait été mieux que Philotas s'en fût tenu à pécher par des propos »).

41. Pour prendre un seul exemple, alors qu'au livre 7, il songe à mesurer une gloire qu'il partage avec ses soldats (7.7.13 : «fallitur qui terminos gloriae nostrae metitur spatio, quod transituri sumus ", «il se trompe celui qui mesure l'étendue de notre gloire d'après l'espace que nous nous apprêtons à traverser »), au livre 9, dans le récit de la campagne contre les Sudraques, il ne songe plus qu'à mesurer une gloire qui lui est propre (9.9.18 : « ego me metior non aetatis spatio, sed gloriae ", « je ne me mesure pas à l'étendue de mon âge, mais à celle de ma gloire »).

42. Ainsi, à propos de "l'affaire Philotas ", J.A.P. Gissel, op. cit., p. 222 : «even though Alexander in the episode itself makes the army do what he wants and even willingly so, the affair is nevertheless placed in the context of growing distance between the king and the Macedonians ", " même si Alexandre dans 
l'épisode amène les armées à faire ce qu'il veut, et même sans y être forcées, l'affaire est néanmoins placée dans le contexte de la distance grandissante qui s'installe entre le roi et les Macédoniens. »

43. Ainsi, pour évoquer un autre type de passage que les discours, à mesure que le récit avance, les marches de l'armée ou l'évocation des blessures d'Alexandre, au lieu de servir uniquement l'héroïsation de celui-ci, soulignent la dépendance des Macédoniens envers leur roi, ainsi que l'affirmation de comportements monarchiques chez celui-ci; cf. O. Devillers, "Analogies et contrastes dans les Historiae Alexandri de Quinte-Curce. Alexandre chez les Scythes et les Sudraques ", à paraître dans M. Mahé-Simon et J. Trinquier éd., Actes de la Journée d'Études sur Quinte-Curce, Paris, ENS, 2010. On songe à l'observation de J.A.P. Gissel, op.cit., p. 215 sur les Historiae : " a work in which biographical and historiographical methodes are combined in such a way that depictions of battles and speeches are used to characterize the figure of Alexander ", " un travail dans lequel les méthodes biographiques et historiographiques sont combinées de telle sorte que les peintures de batailles et les discours servent à caractériser la figure d'Alexandre. »

44. Sur l'influence de la rhétorique sur Quinte-Curce, W. Rutz, "Zur Erzählungskunst des Q. Curtius Rufus ", Aufstieg und Niedergang der römischen Welt, II.32.4, 1986, p. 2352-2353 ; aussi E.I. Mac Queen, «Quintus Curtius Rufus », in: T.A. Dorey éd., Latin Biography, Londres, Routledge \& K. Paul, 1967, p.17-43; O. Devillers, «Quinte-Curce, Historiae Alexandri, VIII-X », dans Silves latines 2009-2010, Paris, Atlande, 2009, p. 49-59.

45. L. Ballesteros-Pastor, op. cit., spéc. p. 34-37.

46. Par ex. W. Heckel, op. cit., p. 69-70 ; E. Baynham, op. cit., p. 52 ; R.H. Martin, Tacitus. Annals V \& VI, Warminster, Aris \& Phillips, 2001, p. 121 ; aussi J. Marincola, op. cit., p. 132.

47. De même, par ex., I. Yakoubovitch, op. cit., p. 30.

\section{AUTEUR \\ OLIVIER DEVILLERS}

Université Bordeaux Montaigne - UMR 2607 Ausonius - ANR Dioneia 